\title{
COMPACTACIÓN INDUCIDA POR EL TRÁFICO DE MÁQUINAS EN SUELO FORESTAL
}

\author{
Jorge Claverie ${ }^{1}$, Roberto Balbuena ${ }^{1}$, Antonino Terminiello ${ }^{1}$, Juan Casado ${ }^{2}$, \\ Eduardo Manghi $^{2}$ \& Daniel Jorajuría ${ }^{1}$
}

\begin{abstract}
RESUMEN
Se realizaron ensayos en campo para evaluar los efectos del tráfico de cosecha y extracción sobre las propiedades físicas del suelo en una plantación de álamos (Populus sp.). Fueron evaluadas: a) zonas sin tránsito vehicular $\mathrm{y}$ b) zonas transitadas por maquinaria de cosecha y extracción. Para ambas situaciones se efectuaron mediciones de resistencia a la penetración, humedad y densidad aparente. La resistencia a la penetración en las zonas transitadas resultó significativamente mayor hasta los 150 $\mathrm{mm}$ de profundidad, siendo el efecto del tránsito detectable hasta los $380 \mathrm{~mm}$. A partir de los $150 \mathrm{~mm}$ ambos tratamientos superaron valores de 1,5 MPa, que reducirían el crecimiento radicular. La densidad en los sectores con tránsito resultó significativamente mayores, siendo en los primeros $80 \mathrm{~mm}$ significativamente menor respecto a los estratos mas profundos para ambos tratamientos. La presencia de capas de elevada densidad aparente influye en la compactación superficial.
\end{abstract}

Palabras clave: tránsito, densidad aparente, resistencia a la penetración

\section{MACHINERY TRAFFIC INDUCED COMPACTION IN FOREST SOIL}

\begin{abstract}
Field tests were carried out with the objective of evaluating the wood harvest and extraction traffic effects on soil physical properties on a commercial poplar plantation (Populus sp.). Two situations were evaluated: a) non-transited zones and b) transited zones by the harvest machinery. Penetration resistance, bulk density and gravimetric moisture were determined on non-transited zones and in the vehicles tracks. Penetration resistance values measured in the vehicles tracks were significantly higher than non transited zones up to $150 \mathrm{~mm}$ depth, and transit effect was detectable up to $380 \mathrm{~mm}$ depth. From $150 \mathrm{~mm}$, both treatments overcame 1.5 MPa values, which would produce root growth reductions. Mean bulk density on transited zones was significantly higher than non-transited zones. Depth analyses values were significantly lower in the first $80 \mathrm{~mm}$ than the lower stratas in each treatment. High bulk density layer had an influence in shallow soil compaction.
\end{abstract}

Key words: traffic, bulk density, penetration resistance

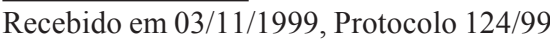

${ }^{1}$ Docente-investigador. Depto. de Ing. Rural. Facultad de Cs. Agrarias y Ftales. UNLP. Av. 60 esq. 119 (1900), La Plata, Argentina. Fone: (54) 221423 6758, Fax: (54) 221425 2346. E-mail: balbuena@isis.com.edu.ar

${ }^{2}$ Becario del Depto. de Ing. Rural. Facultad de Ciencias Agrarias y Frutales. UNLP 


\section{INTRODUCCIÓN}

La cosecha forestal implica el tránsito de maquinaria pesada sobre el suelo que constituye el sustrato en el que crecen los árboles. El abastecimiento a la industria requiere un flujo regular que no puede ser interrumpido cuando las condiciones edáficas no son las óptimas para la traficabilidad. Así, el suelo resulta a veces profundamente alterado, con desplazamiento de las capas superficiales y formación de profundas huellas. Según Taylor \& Gill (1984), la principal fuente de compactación es el tráfico vehicular, el cual puede causar de acuerdo a Jorajuría (1996), disminución del espacio poroso, menor capacidad para retener agua y una mayor impedancia para el desarrollo radicular. Smith \& Dickson (1990), indicaron que la compactación de las capas superficiales está influenciada principalmente por la presión superficial en la zona de contacto rueda-suelo, mientras que la compactación subsuperficial está directamente influenciada por el peso. Al respecto, Håkansson \& Danfors (1981) determinaron que cargas mayores de $60 \mathrm{kN}$ por eje, u 80 a $100 \mathrm{kN}$ para ejes en tandem, provocarían compactación a profundidades superiores a los $400 \mathrm{~mm}$. Sin embargo, la compactación subsuperficial depende no solo del peso del vehículo sino también del número de pasajes que efectúe (Jorajuría \& Draghi, 1997). Gameda et al. (1987) indicaron que la compactación en los horizontes superficiales de suelos agrícolas estuvo más fuertemente emparentada con el número de pasajes que con la presión de contacto ejercida. En tal sentido, Soane et al. (1976) concluyeron que la zona de máxima compactación tiende a aproximarse a la superficie a medida que el número de pasajes aumenta.

Dos parámetros utilizados frecuentemente para cuantificar la compactación del suelo son la resistencia a la penetración y la densidad aparente. Con relación al primero de ellos, Yasin et al. (1993), establecieron una relación lineal entre el índice de cono y el contenido de humedad. Según Threadgill (1982), valores de resistencia a la penetración superiores a 1,5 $\mathrm{MPa}$ implican reducciones en el crecimiento radicular, en tanto que valores de 2,1 a 2,5 MPa actúan como limitantes del mismo. En cuanto a la densidad aparente, Sands et al. (1979), encontraron que incrementos de 1,35 a $1,48 \mathrm{Mg} \mathrm{m}^{-3}$ produjeron reducciones del 10 al $17 \%$ en el rendimiento mientras que valores de 1,60 $\mathrm{Mg} \mathrm{m}^{-3}$ determinaban una reducción del $50 \%$ en el rendimiento de plantaciones de pino en suelos arenosos. Erbach (1986) puntualizó que aún con un cuidadoso procedimiento de toma de muestras y un adecuado número de repeticiones, la sensibilidad de las determinaciones de densidad aparente no excede el valor de $0,05 \mathrm{Mg} \mathrm{m}^{-3}$. A partir de esta propuesta,
Adam \& Erbach (1995) sostuvieron que la profundidad a la cual las diferencias entre la densidad aparente de zonas con y sin tránsito igualan dicho valor, determinaría la profundidad de la labranza necesaria para la remoción de la compactación inducida. El objetivo de este trabajo fue evaluar la compactación inducida por el tráfico de maquinas en la cosecha y extracción de madera.

\section{MATERIAL Y MÉTODOS}

Los ensayos se efectuaron en el establecimiento María Dolores, perteneciente a la empresa Papel Prensa, ubicado en el partido de Alberti, provincia de Buenos Aires, Argentina (34 $50^{\prime}$ $\left.\mathrm{LS} ; 60^{\circ} 30^{\prime} \mathrm{LW} ; 55 \mathrm{msnm}\right)$. Se trabajó en una plantación de Pópulus deltoides, clon Catfish 2. La cosecha se realizó a la edad de 12 años con destino a la industria papelera. El suelo pertenece a la serie Bragado, fase moderadamente bien drenada, taxonómicamente Hapludol típico, Limosa fina, Mixta térmica (Keys to Soil Taxonomy, 1992). El corte fue realizado por un harvester (recogedora de árboles), con orugas de $0,7 \mathrm{~m}$ de ancho y 3,43 m de largo, con un peso total de $196 \mathrm{kN}$. La maquinaria de extracción utilizada se detalla en la Tabla 1.

Dentro de la plantación se distinguieron dos tratamientos: uno correspondiente a zonas transitadas por los vehículos (CT) y otro a zonas no transitadas (ST). En ambas situaciones se establecieron 6 estaciones de muestreo en las cuales se tomaron datos de resistencia a la penetración por medio de un penetrómetro de cono (ASAE S313.2, 1992) en intervalos de $76,2 \mathrm{~mm}$ hasta una profundidad de $686 \mathrm{~mm}$. La densidad aparente se determinó mediante el método del cilindro (Forsythe, 1975), en 5 intervalos de profundidad sucesivos de $80 \mathrm{~mm}$ cada uno. A partir de las muestras de suelo extraídas para la determinación de densidad, se obtuvieron los datos de humedad gravimétrica. El análisis estadístico de los resultados se realizó mediante el análisis de la varianza, cuantificándose las diferencias por medio del test de Tukey.

\section{RESULTADOS Y DISCUSIÓN}

La media de humedad gravimétrica fue de 29,45 y $31 \%$ para el tratamiento con tránsito y sin tránsito, respectivamente, no registrándose diferencias significativas entre ambos. Esto posibilitó comparar su resistencia a la penetración, de acuerdo a lo establecido por Yasin et al. (1993).

La densidad aparente en zonas transitadas fue significativamente mayor que en las no transitadas, al comparar el valor medio de todos los intervalos de profundidad entre

Tabla 1. Maquinaria utilizada en la extracción de madera

\begin{tabular}{|c|c|c|c|}
\hline \multirow[t]{2}{*}{ Vehículo } & \multicolumn{2}{|c|}{ Peso $(\mathrm{kN})$} & \multirow[t]{2}{*}{ Neumáticos } \\
\hline & Peso total & Peso/eje & \\
\hline Tractor $\mathrm{FWA}^{*}$ & 70 & Trasero: 42 & Trasero: $24.5 \times 32$ \\
\hline (Tracción delantera asistida) & & Delantero: 28 & Delantero : $13,9 \times 26$ \\
\hline \multirow[t]{2}{*}{ Camión de 2 ejes } & Vacío: 78 & Vacío: 39 & $11 \mathrm{R} 20$ duales \\
\hline & Cargado: 175 & Cargado: 87,5 & \\
\hline \multirow[t]{2}{*}{ Acoplado de 3 ejes } & Vacío: 58 & Vacío: 19,3 & 11 R 202 ejes duales \\
\hline & Cargado: 225 & Cargado: 75 & \\
\hline \multirow[t]{2}{*}{ Tractor c/garra (2) } & 63 & Trasero: 44,1 & Trasero: $23.1 \times 30$ \\
\hline & & Delantero: 18,9 & Delantero: $6 \times 16$ \\
\hline
\end{tabular}


Tabla 2. Medias de densidad aparente* $\left(\mathrm{Mg} \mathrm{m}^{-3}\right)$ entre tratamientos (ST - zonas no transitadas; $\mathrm{CT}$ - zonas transitadas por los vehiculos) para todo el perfil y para cada profundidad

\begin{tabular}{lcccccc}
\hline Tratamiento & \multicolumn{5}{c}{ Profundidad - mm } \\
\cline { 2 - 6 } & General & $0-80$ & $80-160$ & $160-240$ & $240-320$ & $320-400$ \\
\hline ST & $1,17 \mathrm{a}$ & $0,76 \mathrm{a}$ & $1,23 \mathrm{a}$ & $1,36 \mathrm{a}$ & $1,36 \mathrm{a}$ & $1,16 \mathrm{a}$ \\
CT & $1,25 \mathrm{~b}$ & $0,94 \mathrm{a}$ & $1,36 \mathrm{~b}$ & $1,36 \mathrm{a}$ & $1,36 \mathrm{a}$ & $1,23 \mathrm{a}$ \\
\hline * & &
\end{tabular}

* Valores seguidos de la misma letra inicial no tienen diferencias significativas al $5 \%$ de probabilidades del test de Tukey

ambos tratamientos. El análisis de los intervalos de profundidad entre tratamientos no arrojó diferencias significativas, excepto para el intervalo de 80 a $160 \mathrm{~mm}$, en el que el tratamiento con tránsito alcanzó el límite inferior indicado por Sands \& Bowen (1979), como causante de reducción de rendimiento (Tabla 2).

En la Figura 1 se observan las diferencias en valores absolutos entre zonas transitadas y no transitadas

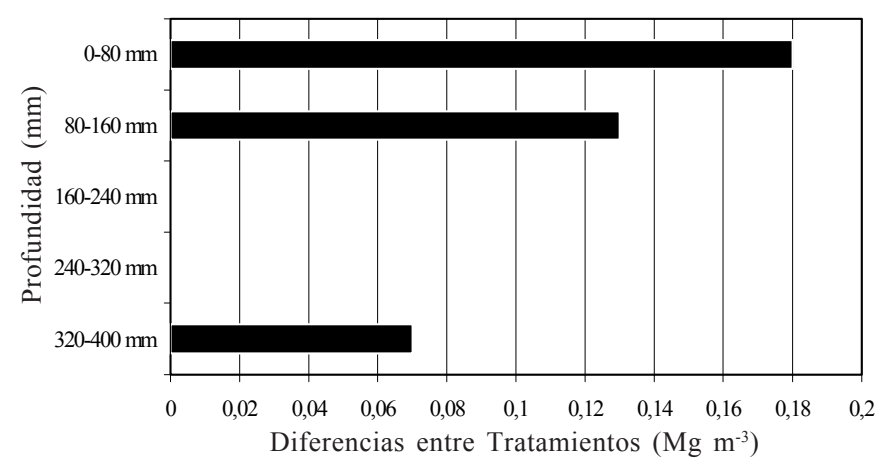

Figura 1. Diferencias entre densidad aparente de zonas con y sin tránsito

Las diferencias entre zonas con y sin tránsito se igualan tanto en el intervalo de 160 a $240 \mathrm{~mm}$ como en el de 240 a 320 . Lo observado podría deberse al estado de densificación natural del perfil del suelo en los mencionados estratos previo al pasaje de. Entre los 320 y $400 \mathrm{~mm}$ se alcanza un valor de $0.07 \mathrm{Mg} \mathrm{m}^{-3}$ que justificaría, de acuerdo a Adam \& Erbach (1995), la realización de una labranza a esta profundidad. En este sentido, debido a los elevados esfuerzos de tracción que implicaría el laboreo, existiría la posibilidad de que el peso adherente del tractor represente un factor concurrente a la inducción de compactación, en la medida que la profundidad de trabajo resulte insuficiente para disminuir las tensiones ejercidas al suelo por el propio tráfico durante la labor.

Tanto el peso por eje del camión como del acoplado cargados superan los valores propuestos por Smith \& Dickson (1990) y Håkansson \& Danfors (1981), como límites para mantener la compactación a nivel superficial, por lo cual sería esperable un aumento en la compactación aún por debajo de los $400 \mathrm{~mm}$. A la vez, el tránsito repetido sobre la zona densificada entre 160 y $240 \mathrm{~mm}$ podría en parte ser determinante de los incrementos en la compactación del suelo a nivel superficial (Soane et al., 1976;
Gameda et al., 1987), a lo cual contribuiría la presión superficial en la zona de contacto rueda suelo, tanto del tractor como del acoplado (Smith \& Dickson, 1990).

En relación al índice de cono (Tabla 3), los valores determinados en zonas transitadas resultaron significativamente mayores que los del testigo hasta los $150 \mathrm{~mm}$ de profundidad, siendo el efecto del tránsito detectable hasta el estrato de 381 a $457 \mathrm{~mm}$. Los incrementos porcentuales con respecto al tratamiento sin tránsito fueron de $62,6,26,7,13,7$ y $6,4 \%$ para los estratos comprendidos entre $152 \mathrm{~mm}$ y $381 \mathrm{~mm}$ respectivamente. Esto podría explicarse a partir del estado del suelo previo al pasaje de los vehículos, dado que a partir de los $150 \mathrm{~mm}$ de profundidad, el testigo registró valores cercanos o superiores a 1,5 MPa, en tanto que en las zonas transitadas se registraron valores superiores a 2,1 MPa desde el primer intervalo de profundidad, que implicarían reducciones en el desarrollo radicular según Threadgill (1982), determinándose registros similares para el testigo a partir de los $228 \mathrm{~mm}$ de profundidad.

\section{CONCLUSIONES}

1. La presencia de estratos densificados en el perfil influye en la compactación superficial.

2. La alteración de las propiedades físicas del suelo inducida por el tráfico repetido justifica la realización de laboreo profundo.

\section{REFERENCIAS BIBLIOGRÁFICAS}

ADAM, K.M.; ERBACH, D.C. Relationship of tire sinkage depth to depth of soil compaction. Transactions of the ASAE, St. Joseph, MI, v.38, n.4, p.1011- 1016, 1995.

ASAE S 313.2: Soil cone penetrometer. In: American Society of Agricultural Engineers (ed.). ASAE Standards. St. Joseph, MI, 1992. p.611.

ERBACH, D.C. Farm equipment and soil compaction. SAE Paper No 86-0730, St. Joseph, MI, p.170-176, 1986.

FORSYTHE, W. Manual de laboratorio de física de suelos. San José: Instituto Iberoamericano de Ciencias Agrícolas, 1975. p.39-45.

GAMEDA, S.; RAGHAVAN, G.S.V.; MCKYES, E.; THÉRIAULT, R. Subsoil compaction in a clay soil. I. Cumulative effects. Soil and Tillage Research, Amsterdam, v.10, p.113-122, 1987.

Tabla 3. Medias de índice de cono (MPa)* por intervalos de profundidad (mm) (ST - zonas no transitadas; CT - zonas transitadas por los vehiculos)

\begin{tabular}{lcccccccccc}
\hline Tratamiento & \multicolumn{10}{c}{ Profundidad - mm } \\
\cline { 2 - 10 } & 0 & $0-76$ & $76-152$ & $152-228$ & $558-305$ & $305-381$ & $381-457$ & $457-534$ & $534-610$ & $610-686$ \\
\hline ST & $0,63 \mathrm{a}$ & $1,09 \mathrm{a}$ & $1,41 \mathrm{a}$ & $1,82 \mathrm{a}$ & $2,17 \mathrm{a}$ & $2,48 \mathrm{a}$ & $2,64 \mathrm{a}$ & $3,60 \mathrm{a}$ & $4,63 \mathrm{a}$ & $5,44 \mathrm{a}$ \\
$\mathrm{CT}$ & $1,28 \mathrm{~b}$ & $2,31 \mathrm{~b}$ & $2,25 \mathrm{~b}$ & $2,96 \mathrm{a}$ & $2,75 \mathrm{a}$ & $2,82 \mathrm{a}$ & $2,81 \mathrm{a}$ & $3,20 \mathrm{a}$ & $3,98 \mathrm{a}$ & $4,65 \mathrm{a}$ \\
\hline * Valores seguidos de la misma letra inicial no tienen diferencias significativas al 5\% de probabilidades del test de Tukey
\end{tabular}


HÅKANSSON, Y; DANFORS, B. Effects of heavy traffic on soil conditions and crop growth. In: PROCEEDINGS OF INTERNATIONAL CONFERENCE OF THE INTERNATIONAL SOCIETY FOR TERRAIN-VEHICLE SYSTEMS, 7, Alberta, v. Y, 1981.p.239-253.

JORAJURÍA, D. El tráfico vehicular, la compactación y la sostenibilidad de la producción de madera. In: Actas del Primer Seminario de Actualización en Sistemas de Cosecha y Transporte Forestal, 1996, Eldorado. p.45-50.

JORAJURÍA, D; DRAGHI, L. The distribution of soil compaction with depth and the response of a perennial forage crop. Journal of Agricultural Engineering Research, London, v. 66, p.261-265, 1997

KEYS TO SOIL TAXONOMY. USDA. Soil Survey Staff. AID. SMSS. Technical Monograph $N^{\circ} 19$, Washington D.C., Virginia Polytechnic Institute and State University, 1992. 754p.

SANDS, R. ; GEACEN, E.L.; GERARD, C.J. Compaction of sandy soils in radiata pine forests. 1. A penetrometer study. Australian Journal of Soil Research, Sidney, v.17, p.101-113, 1979.
SMITH, D.; DICKSON, J. Contributions of vehicle weight and ground pressure to soil compaction. Journal of Agricultural Engineering Research, London, v.46,n.1, p.13-29, 1990.

SOANE, B.D.; KENWORTHY, G.; PIDGEON, J.D. Soil tank and field studies of compaction under wheels. PROCEEDINGS OF INTERNATIONAL CONFERENCE OF SOIL AND TILLAGE RESEARCH, 7, Estocolmo, 45, Paper 48, 1976.

TAYLOR, J; GILL, W. Soil compaction: State-of-art report. Journal of Terramechanics, Oxford, v.21, n.3, p.195-213, 1984.

THREADGILL, E.D. Residual tillage effects as determined by cone index. Transactions of the ASAE, St. Joseph, MI, v.25, p.859-863, 1982.

YASIN, M.; GRISSO R.D.; BASHFORD L.L.; JONES, A.J.; MIELKE, L.N. Normalizing cone resistance values by covariance analysis. Transactions of the ASAE, St. Joseph, MI, v.36, n.5, p.1267-1270, 1993. 